\title{
Opportunity and challenge: ten years of proteomics in China
}

\author{
LENG FangWei \\ Institute of Biophysics, Chinese Academy of Sciences, Beijing 100101, China
}

Received April 19, 2012; accepted May 20, 2012

Citation: Leng F W. Opportunity and challenge: ten years of proteomics in China. Sci China Life Sci, 2012, 55: 837-839, doi: 10.1007/s11427-012-4372-1

Proteomics is a science that focuses on the global analysis of protein expression and function in organisms. Although it is still a relatively new field, it has become increasingly important for life science research [1-3]. Since introduced in China in 1997, proteomics has developed rapidly in the past ten years in China with the support of the Chinese central and local governments. As a core part of the first Major Scientific Research Program, it is now a major research area in China. There emerge many excellent Chinese scientists, who have conducted much remarkable work in this filed [4-8]. In recent years, many research laboratories, facilities and centers have been established for proteomics, including the Research Center for Proteome Analysis, the Institutes of Biomedical Sciences, Proteomics Key Lab, and Beijing Proteome Research Center (BPRC). Beijing Proteome Research Center (BPRC) is the first proteomics institute established in China, and has become a top proteomics institute. The Chinese Human Liver Proteome Project (CNHLPP) led by BPRC established the first dataset of the human liver proteome (HLP), and also the dataset of human liver transcriptome (HLT) which is found directly associated with HLP [9]. These achievements will greatly enhance understanding of liver diseases such as non-alcoholic steatohepatitis, HBV infection, and hepatic cancers, and contribute to the development of therapeutics for these diseases. To display the progress of proteomics in China, the first issue of Science China Life Sciences (2011) organizes a special issue with eight research papers in proteomics provided by Chinese scientists, covering mitochondrial proteome, human liver proteome, secretome extraction methods, immobilized

email: fangweihaha7@gmail.com enzyme reactors and so on.

The first paper entitled "Proteomic survey towards the tissue-specific proteins of mouse mitochondria" by Wang et al. [10] focuses on tissue-specific mitochondrial proteins. Researchers integrated multiple approaches to systematically analyze proteins that are localized in mouse mitochondria, finding that the tissue-specific mitochondrial proteins were widely distributed among the mouse tissues. This paper provides a set of data for exploring the biological significance of tissue-specific mitochondrial proteins. It will be helpful for understanding mitochondrial physiology and pathology. The second paper is entitled "Antigenically dominant proteins within the human liver mitochondrial proteome identified by monoclonal antibodies" by Ju et al. [11]. This paper also focuses on mitochondrial proteome. Researchers defined six proteins as antigenically dominant proteins, and tested the monoclonal antibodies for their ability to deplete the recognized protein from liver tissue lysates. These studies provide strong evidence that depletion of dominant proteins from liver mitochondrial samples is possible.

The next paper entitled "Toward a high resolution 2-DE profile of the normal human liver proteome using ultra-zoom gels" by Mi et al. [12] focuses on the aforementioned human liver proteome (HLP). In this paper, researchers used ultra-zoom gels to perform proteomic analysis of normal human livers, and constructed a reference map of the proteins of human normal liver. It will contribute to comparative proteomic research of the liver proteome. Ultrafiltration, precipitation, and dialysis are three major extraction methods for comprehensive recovery of secreted proteins [13-15]. The paper entitled "Comparison of alter- 
native extraction methods for secretome profiling in human hepatocellular carcinoma cells" by Cao et al. [16] compares the merits and shortcomings of these three extraction methods without bias for the first time, offering a reference for the selection of secretome extraction methods.

The fifth paper is entitled " $\alpha-2-H S$-glycoprotein is a potential marker predicting hepatitis B e antigen seroconversion in patients with chronic hepatitis B during treatment with PEGylated interferon alfa-2b" by Ma et al. [17]. Researchers used two-dimensional electrophoresis (2-DE)based proteomic strategies to identify potential serum markers predicting hepatitis $\mathrm{B}$ e antigen ( $\mathrm{HBeAg}$ ) seroconversion during IFN treatment in CHB patients. The study suggests that dynamic changes in the serum level of $\alpha$-2-HS-glycoprotein may be a potential early marker predicting $\mathrm{HBeAg}$ seroconversion in IFN treatment for CHB.

Matrix-assisted laser desorption/ionization time-of-flight mass spectrometry (MALDI-TOF MS) is an efficient method for classifying and identifying bacteria $[18,19]$. The paper entitled "Whole-cell matrix-assisted laser desorption/ionization time-of-flight mass spectrometry for rapid identification of bacteria cultured in liquid media" by Zhou et al. [20] explores the use of MALDI-TOF MS analysis for rapid identification of cells cultured in liquid media, and reveals the great potential for using MALDI-TOF MS as a more rapid method for direct bacterial identification. High efficiency enzymatic digestion is crucial for shot gun proteomics, and it is thus not strange that great attention has been paid to various immobilized enzyme reactors (IMERs) for the realization of high throughput digestion in proteomics [21,22]. The paper entitled "A novel organic-inorganic hybrid monolith for trypsin immobilization" by $\mathrm{Wu}$ et al. [23] reports a novel organic-inorganic hybrid monolith based IMER, having superior digestion activity and stability.

Transporters are often membrane proteins, playing important roles in compound exchange and metabolic flux control [24]. The last paper entitled "TSdb: A database of transporter substrates linking metabolic pathways and transporter systems on a genome scale via their shared substrates" by Zhao et al. [25] presents the first curated transporter substrate database (TSdb), which is a high-quality resource of transporters and transporter substrates mapped to KEGG ligand compound IDs. It is hoped that this database will facilitate the study of transporter systems.

With constant increase of support and attention, proteomics has achieved great development in the past decade in China. Many research laboratories, facilities and centers have been established. Many important proteomics projects led by Chinese scientists such as CNHLPP are under way. Many techniques and strategies for proteomics are developed and improved constantly [26-30]. It is believed that proteomics in China will become increasingly widespread in the future. This special issue may contribute to the development of proteomics in China.
1 Wilson R, Whitelock J M, Bateman J F, et al. Proteomics makes progress in cartilage and arthritis research. Matrix Biol, 2009, 28: $121-128$

2 Ruiz-Romero C, Blanco F J. Proteomics role in the search for improved diagnosis, prognosis and treatment of osteoarthritis. Osteoarthritis Cartilage, 2010, 18: 500-509

3 Mobasheri A. Applications of proteomics to osteoarthritis, a musculoskeletal disease characterized by aging. Front Physiol, 2011, 2: 108

4 Bai H, Wang X Y, Cao Y H, et al. Expression profiling of rice chloroplast proteins during growth and development. Prog Biochem Biophys, 2010, 37: 988-995

5 Ouyang Q, Hu R C, Dai A G, et al. Proteomics research focus on the affection of smoking to D4-GDI expression in lung tissue and the relationship with chronic obstructive pulmonary disease. Prog Biochem Biophys, 2010, 37: 1204-1211

6 Zhu Y M, Xiu Q, Dong L R, et al. Identification of proteins associated with let-7a in gastric carcinoma cell line SGC-7901 by proteomics. Prog Biochem Biophys, 2011, 38: 441-448

7 Lan J P, Li L Y, Jia L, et al. Expression profiling of chloroplast-encoded proteins in rice leaves at different growth stages. Prog Biochem Biophys, 2011, 38: 652-660

8 Deng H, Zhang Y L, Zhong B Y, et al. Clinical application and identification of proteomics in colonic mucosa of sub-health people with constipation. Prog Biochem Biophys, 2011, 38: 1043-1051

9 He F C. At a glance: Proteomics in China. Sci China Life Sci, 2011, 54: 1-2

10 Wang Y, Sun H D, Ru Y W, et al. Proteomic survey towards the tissue-specific proteins of mouse mitochondria. Sci China Life Sci, 2011, 54: 3-15

$11 \mathrm{Ju}$ Y F, Yang J J, Liu R, et al. Antigenically dominant proteins within the human liver mitochondrial proteome identified by monoclonal antibodies. Sci China Life Sci, 2011, 54: 16-24

12 Mi W, Liu X, Jia W, et al. Toward a high resolution 2-DE profile of the normal human liver proteome using ultra-zoom gels. Sci China Life Sci, 2011, 54: 25-33

13 Diehl H C, Stuhler K, Volmer M W, et al. A catalogue of proteins released by colorectal cancer cells in vitro as an alternative source for biomarker discovery. Proteomics Clin Appl, 2007, 1: 47-61

14 Trost M, Wehmhöner D D, Kärst U, et al. Comparative proteome analysis of secretory proteins from pathogenic and nonpathogenic Listeria species. Proteomics, 2005, 5: 1544-1557

15 Wu C C, Cheng H C, Chen S J, et al. Identification of collapsin response mediator protein-2 as a potential marker of colorectal carcinoma by comparative analysis of cancer cell secretomes. Proteomics, 2008, 8: 316-332

16 Cao J, Shen C P, Zhang J, et al. Comparison of alternative extraction methods for secretome profiling in human hepatocellular carcinoma cells. Sci China Life Sci, 2011, 54: 34-38

17 Ma H, Wang J H, Guo F, et al. $\alpha$-2-HS-glycoprotein is a potential marker predicting hepatitis $\mathrm{B}$ e antigen seroconversion in patients with chronic hepatitis B during treatment with PEGylated interferon alfa-2b. Sci China Life Sci, 2011, 54: 39-47

18 Ryzhov V, Fenselau C. Characterization of the protein subset desorbed by MALDI from whole bacterial cells. Anal Chem, 2001, 73: 746-750

19 Warscheid B, Fenselau C. A targeted proteomics approach to the rapid identification of bacterial cell mixtures by matrix-assisted laser desorption/ionization mass spectrometry. Proteomics, 2004, 4: 2877-2892

20 Zhou N, Wang N, Xu B, et al. Whole-cell matrix-assisted laser desorption/ionization time-of-flight mass spectrometry for rapid identification of bacteria cultured in liquid media. Sci China Life Sci, 2011, 54: 48-53

21 Li Y, Yan B, Deng C H, et al. Efficient on-chip proteolysis system based on functionalized magnetic silica microspheres. Proteomics, 2007, 7: 2330-2339

22 Slysz G W, Lewis D F, Schriemer D C. Detection and identification of sub-nanogram levels of protein in a nanoLC-trypsin-MS system. J 
Proteome Res, 2006, 5: 1959-1966

23 Wu S B, Ma J F, Yang K G, et al. A novel organic-inorganic hybrid monolith for trypsin immobilization. Sci China Life Sci, 2011, 54: 54-59

24 Ren Q H, Chen K X, Paulsen I T. TransportDB: a comprehensive database resource for cytoplasmic membrane transport systems and outer membrane channels. Nucleic Acids Res, 2007, 35: D274-D279

25 Zhao M, Chen Y M, Qu D C, et al. TSdb: A database of transporter substrates linking metabolic pathways and transporter systems on a genome scale via their shared substrates. Sci China Life Sci, 2011, 54: 60-64

26 Sun R X, Dong M Q, Chi H, et al. ECD/ETD-based tandem mass spectrometry in proteomics. Prog Biochem Biophys, 2010, 37: 94-102

27 Mi W, Wang J. Development of protein quantification based on ICP-MS. Prog Biochem Biophys, 2010, 37: 224-229

28 Sun HC, Zhang J Y, Liu H, et al. Algorithm development of de novo peptide sequencing via tandem mass spectrometry. Prog Biochem Biophys, 2010, 37: 1278-1288

29 Liu W, Xie H W. Potential drug target discovery based on bioinformatics methods. Prog Biochem Biophys, 2011, 38: 11-19

30 Zhang W, Zhang J Y, Liu H, et al. Development of algorithms for mass spectrometry-based label-free quantitative proteomics. Prog Biochem Biophys, 2011, 38: 506-518

Open Access This article is distributed under the terms of the Creative Commons Attribution License which permits any use, distribution, and reproduction in any medium, provided the original author(s) and source are credited. 